\section{Magnetic Induction in a Supra-Conducting Lead Crystal}

IN our last note ${ }^{1}$ we showed the relation between magnetic induction and field strength in the case of a polycrystalline lead rod. We now report shortly on analogous experiments carried out with a lead single crystal.

The measurements were carried out at various temperatures with the second method that we described, the magnetic moment of the supraconductor being determined in a constant field. One may conclude from Fig. 1, in which the results of our measurements at $4 \cdot 24^{\circ} \mathrm{K}$. are shown (full curve), that the destruction of supra-conductivity occurs in almost the same way in single crystals and in poly. crystalline lead. The sudden rise of the induction begins almost exactly at the same critical field strength $H_{k}$ as in polycrystalline material ; however, in single crystals the transition region, that is, the field interval in which the transition from one state to the other begins and terminates, is considerably narrower, extending over merely 7 gauss. In the transition region, the induction is observed to rise slightly with time in a constant magnetic field.

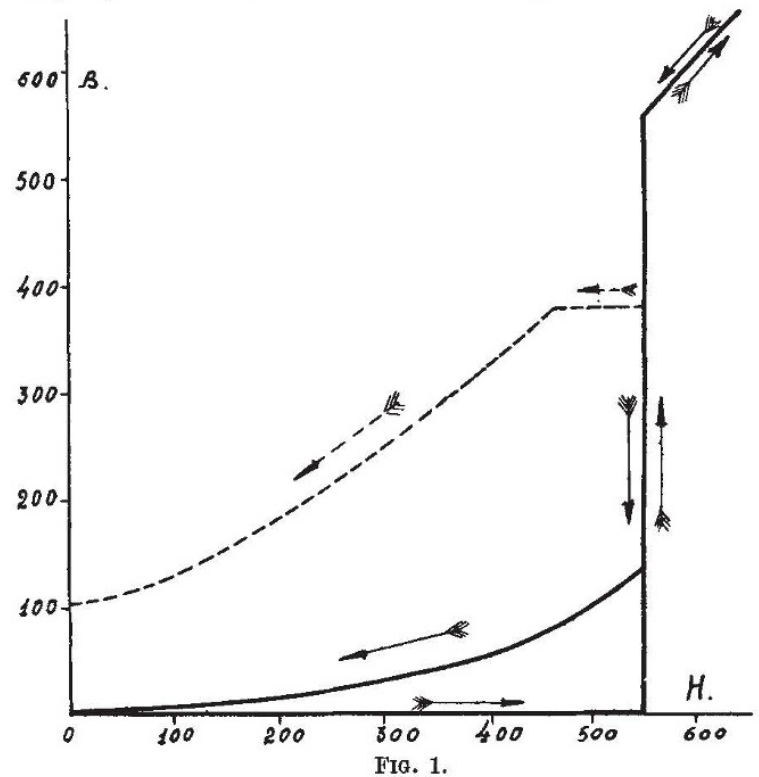

However, the reverse process, the appearance of supra-conductivity on moving from strong fields to fields less than $H_{k}$, is found to be different for single crystals and polycrystalline rods. For comparison, the dotted curve on the same figure shows the relation between $B$ and $H$ for polycrystalline lead. It appeared that in this transition the induction followed a change in the field strength slowly, the process depending strongly on time. The dependence on time was particularly strong in the transition region of the field strength-the same field strengths as those at which the supra-conductivity was destroyed-where even after waiting for half an hour we did not succeed in reaching a state of equilibrium, the induction still changing with noticeable velocity. As the period of our experiments was limited by the speed with which the helium evaporated, the curves which we obtained in decreasing magnetic fields do not correspond to the equilibrium state. On decreasing the field strength to zero a residual magnetisation is observed, which in different experiments varied between 0 and 2 per cent of the maximum of $B$ in the transition region. It was found that the residual magnetisation sank noticeably with time.

The strong dependence on time and the small hysteresis in single erystals have convinced us that all states of a supra-conductor with an induction differing from zero are unstable. These experiments again confirm the concept of two phases, an ordinary and a supra-conducting phase with an induction equal to zero. The transition from the supra-conducting to the ordinary phase occurs rapidly, whereas the reverse transition takes place more slowly. It is interesting to note that the latter process occurs so gradually that a fairly sensitive telephone in connexion with an amplifier is insufficient to detect any spontaneous changes in the induction.

G. N. RJabinin.

L. W. ShubNikow.

Ukrainian Physico-technical Institute, Kharkov.

Nov. 25.

${ }^{2}$ G. N. Rjabinin and L. W. Shubnikow, Nature, 134, 286, Aug. 25, 1934.

\section{Symbols for Chromosome Numbers}

WHILE in entire agreement with Prof. Gates's view ${ }^{1}$ that a convention should be agreed upon to distinguish between the basic and the haploid chromosome number, I am not convinced that the introduction of a new symbol is necessary. The use of $x$ to indicate the basic number, leaving $n$ for the haploid number, has been the standard practice in this laboratory for the last three years ${ }^{2}$. In spite of Prof. Gates's fears, no confusion seems to arise from its use. I may also point out that a Greek letter has the grave practical disadvantage of being troublesome to print and impossible to type on an ordinary machine.

\section{BRENHILDA Schafer,}

Librarian.

John Innes Horticultural Institution, Merton Park, London, S.W.19. Jan. I.

1 NATURE, 134, 1011, Dec. 29, 1934

"See Darlington, "Recent Advances in Cytology", p. 61 (1932) Sansome and Philp, "Recent Advances in Plant Genetics", p. 165 (1932); Crane and Lawrence, "The Genetics of Garden Plants", p. 28 (1934).

\section{Publication of Nomina Nuda}

WE wish to support the appeal of Sir Sidney Harmer in NaTuRE of December 22 (p. 973) for the suppression of nomina nuda by editors of scientific publications.

Editors, however, cannot always be expected to recognise the character of names which, like the one that has caused Sir Sidney Harmer's protest, may perhaps be called nomina seminuda. We would further appeal, therefore, to writers of zoological papers to avoid using names that are not formally introduced according to the rules of zoological nomenclature.
C. Tate Regan,

Director.

W. T. Calman, Keeper of Zoology.

\section{N. D. RILEY,}

Keeper of Entomology. W. D. LANG, Keeper of Geology.
British Museum (Natural History), London, S.W.7. Jan. 7. 